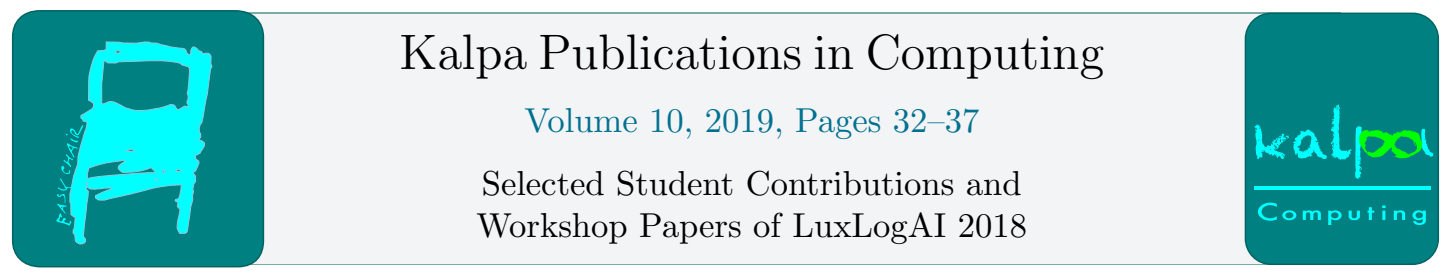

\title{
An Ontology for Transportation System
}

\author{
Durgesh Nandini and Gautam Kishore Shahi \\ Dipartimento di Ingegneria e Scienza dell'Informazione (DISI), \\ University of Trento, Italy \\ durgeshnandini16@yahoo.in, gautamshahi16@gmail.com
}

\begin{abstract}
In this work, we present a domain ontology for Transportation System. We have developed an ontology for a semantics-aware transportation system from the perspective of a traveler user, capable of answering general competence queries like the nearest bus stop to a particular place, the nearest parking slots available, etc. We have studied the transportation system of some of the big cities of the world and have tried to come up with a vocabulary that can be applied to any city with little modifications. This vocabulary is further aligned with an upper-level ontology to have a common starting point.
\end{abstract}

\section{Introduction}

The underlying functioning of an ontology and the mechanism of processing data in a machine readable format makes an ontology an important artifact of the semantic web. Domain ontologies provide a controlled vocabulary of concepts, syntax and semantics that help machine and people communicate.

Information available on current transportation systems is highly unstructured and unorganized [1]. The unstructured representation of transportation system makes it hard to extract meaningful information. Often, a user has to visit several web portals to acquire the required information; it becomes worse in case of a new user who has never visited a city before.

When considering the functioning of the transportation system, a user may be one who has to travel from one place to another (a traveler) or the one who helps a traveler to travel (driver, ticket officer, etc.). Choosing the best way to move from one place to another for a traveler may involve different filters: modes of transportation available, schedule of a public vehicle, parking space availability, safety concerns, etc. On the other hand, for a service provider, various statistics may be of concern, for example, number of passengers travelling, the amount of money collected from tickets, crime rates, number of accidents reported, etc.

The focus of this work is to build an ontology from a traveler's perspective to represent the transportation system. This, in turn, achieves answering user queries in a straightforward and less time-consuming manner. The ontology inherently provides a simple vocabulary for describing a generic transportation system. The vocabulary provides information that a traveler may need and plan his journey accordingly.

The approach followed to build the ontology is by first constructing a set of competence queries and then finding ways to implement them. The competence queries are related to the

C. Benzmüller, X. Parent and A. Steen (eds.), LuxLogAI 2018 (Kalpa Publications in Computing, vol. 10), pp. $32-37$ 
daily movement of a user and related statistics. The competence queries are:

1. At a given bus stop, what is the schedule for buses along with their direction (route number)?

2. What is the crime rate in a particular mode of transportation?

3. What is the list of train stations in a city?

4. What is the parking space availability for a vehicle type at a given street?

5 . What is the number of cycles available for hire?

6 . What is the congestion charge in a given area at a given time?

Remaining sections of the paper are organized as follows: Section 2 discusses Related Works, Section 3 discusses Methodology used for ontology design, Section 4 discusses Implementation \& Experimental Results, and Section 5 discusses Conclusion and Future Work.

\section{Related Works}

Several studies have been done in the area of the ontology-based transportation system. All the related works have been discussed below.

Becker \& Smith [2] defined the representational framework and ontological basis for comprehensive modeling and provide a solution to multimodal transportation planning and scheduling problems. They extended the core framework of Ozone scheduling ontology to formulate the characteristics and constraints for their multi-modal transportation planning and scheduling, and then used the extended framework as a basis for elaborating concepts of particular relevance to transportation planning and scheduling.

Timpf [3] built two types of ontology-one from the user perspective and another from transportation system perspective and showed that ontology from the perspective of the user is a part of transportation system perspective, thus leading to the idea of nested ontology.

Wang, Ding, and Jiang [4] developed an ontology public transport query system to provide well and timely information service. They have used an improved public transport transfer query algorithm, which considers both customer's needs and road conditions. They tried to solve the problem of same station name at different places to find the shortest distance between two places using a spatial radius search algorithm.

Niaraki and Kim [5] provided an ontology-based abstract model for user-centric personalized route planning system using Analytic Hierarchical Process (AHP) to calculate the shortest distance between any two nodes by using personalized impedance modeling. They created domain specific ontology and road segment ontology to give a personalized route to the user. The methodology is able to deal with route planning only.

Houda et al. [6] developed a domain based transportation system for passenger planning system. The system supports a multi-model journey pattern, which helps the user to find the most convenient path from one position to another with several possible options. Choice of the user depends on interesting journey patterns, shopping journey pattern, etc. The system is limited to the roadways and railways only.

Olivera, Bacha, Mnasser, and Abed [7] provide a user-interface transportation system based on Model-driven Architecture (MDA) approach with features of information filtering, personalized information query, auto-fills forms. It provides a personalized travel planning but is limited to roadways and railways only. To use this approach, a user requires having a complete knowledge of the transport domain. Another limitation is that, this approach is only for journey planning.

The above mentioned related works give an idea about a domain based transport ontology to deal with travel planning between two locations. The related works propose systems only 
for roadways or railways. However, they do not deal in answering user queries related to multimodal mobility, parking space availability, details about crime rate etc.

In this paper, An ontology for Transportation System is presented for a semantics aware transportation system which covers all modes of transports and it is able to deal with trip planning as well as to answer the queries related to safety, cost-effective planning etc.

\section{Methodology for Ontology Development}

The transportation system of different cities - both metropolitan and small cities were studied. Some of the cities surveyed were, for instance, London, Berlin, Paris, Koblenz, Trento, Auckland, Ohio etc. It was observed that the transportation system of London was divided into multi-mode transportation facilities: waterway, roadway, airway, etc. and within each mode of transport there were different options available. We have followed the DERA( Domain, Entity, Relation, Attribute) methodology [8, 9, 10] to construct the ontology and enabled ontology reusue by following the Metadata for Ontology Description and Publication (MOD) [11] standards.

DERA facets consist of Domain, Entity, Relation, and Attribute. The domain of our ontology is transport.Aligning with DERA gives our ontology a defined structure. The assignation shows that our ontology has a clear domain, has a correct set of entities and shows relationships between these entities.

After the construction of the hierarchy, all the entities were aligned with the Descriptive Ontology for Linguistic and Cogninitive Engineering (DOLCE) [12], one of the top-level ontology. Alignment with an upper ontology enriched our ontology to be semantically interoperable among a large number of domain ontologies by having a similar starting point.

\section{Implementation \& Experimental Results}

We use Protege-5.1.0 [13] to build our ontology because it is a free, open-source editor and framework and it supports the latest OWL 2 Web Ontology Language and RDF specifications from the World Wide Web Consortium. After refinement, our ontology comprised of 46 classes, 42 distinct data properties, and 8 distinct object properties. The Figure 1 represent the class inferred hierarchy.

Extended Entity-Relationship model (EX-ER model) describes inter-related things of interest in a specific domain of knowledge. An EX-ER model is composed of entity types (which classify the things of interest) and specifies relationships that can exist between instances of those entity types. Figure 2 shows the Extended Entity-Relationship Model of the transport ontology.

For any ontology to be consistent, it is important that there are no contradicting concepts and relations. Mutual compatibility of classes, attributes and relations should also be kept into account. To check the consistency of our ontology we have used HermiT as a reasoner and to validate SPARQL queries has been performed to retrieve information, two of them has been shown here for demonstration purpose in Figure 3 and Figure 4. 

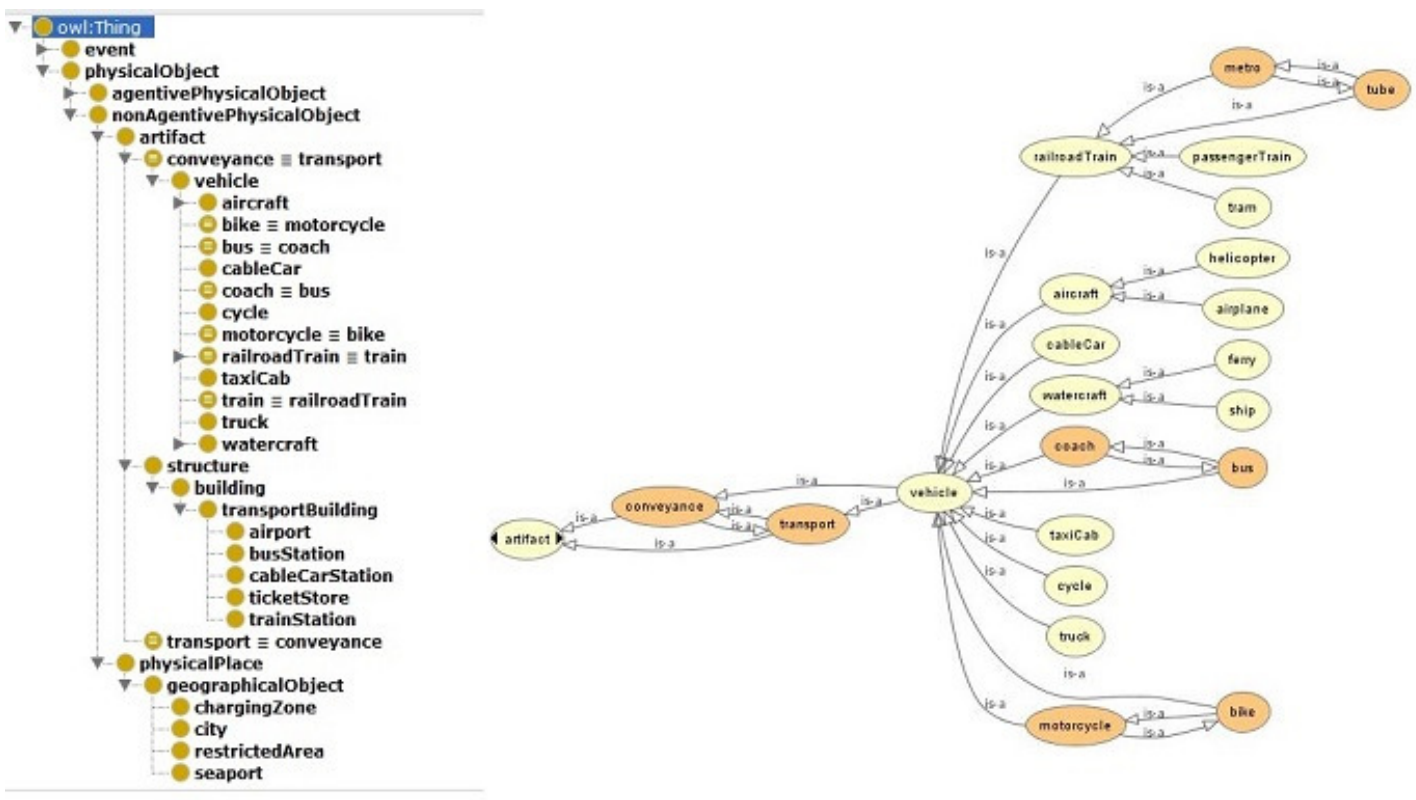

Figure 1: Class inferred hierarchy

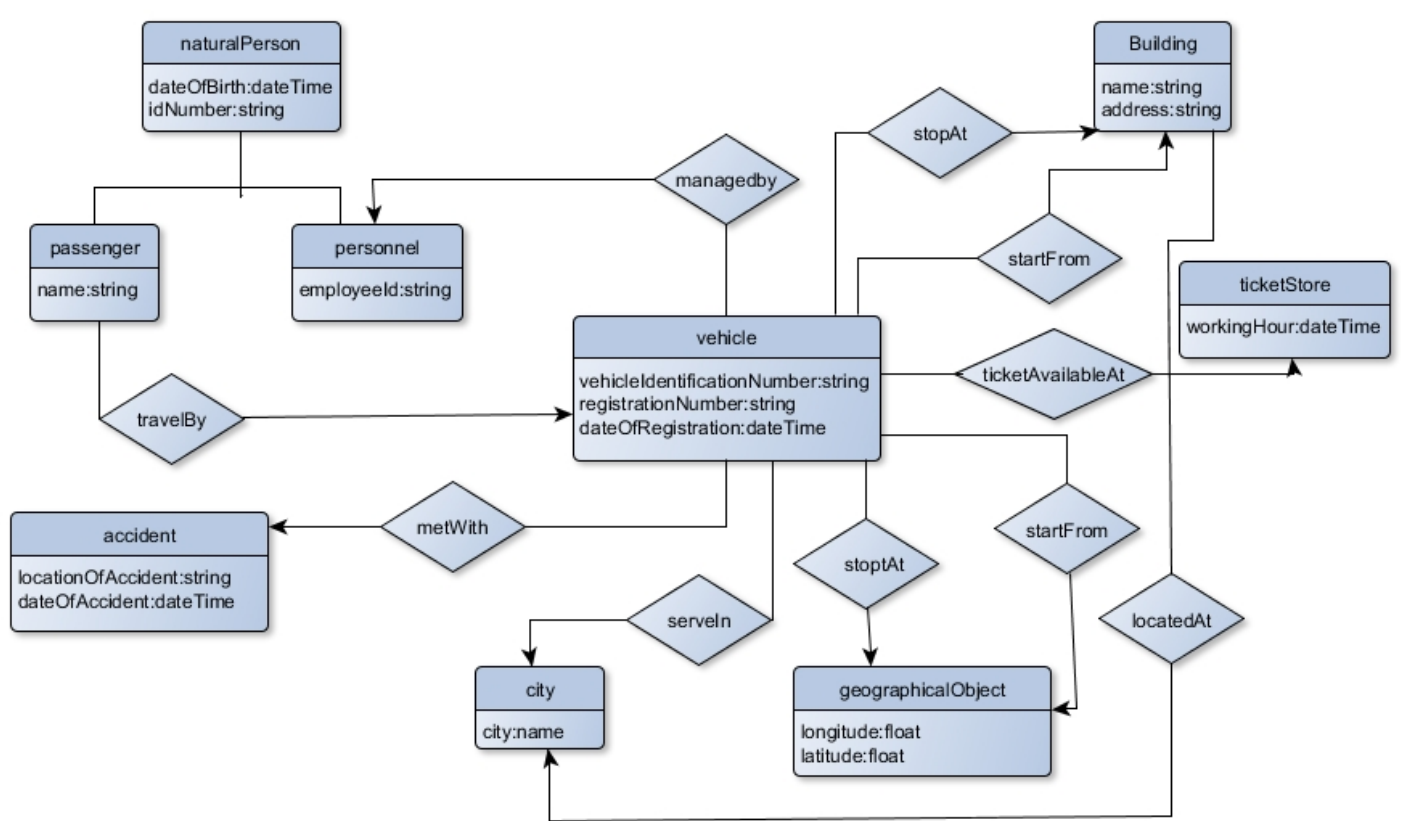

Figure 2: Extended Entity-Relationship Model Diagram 


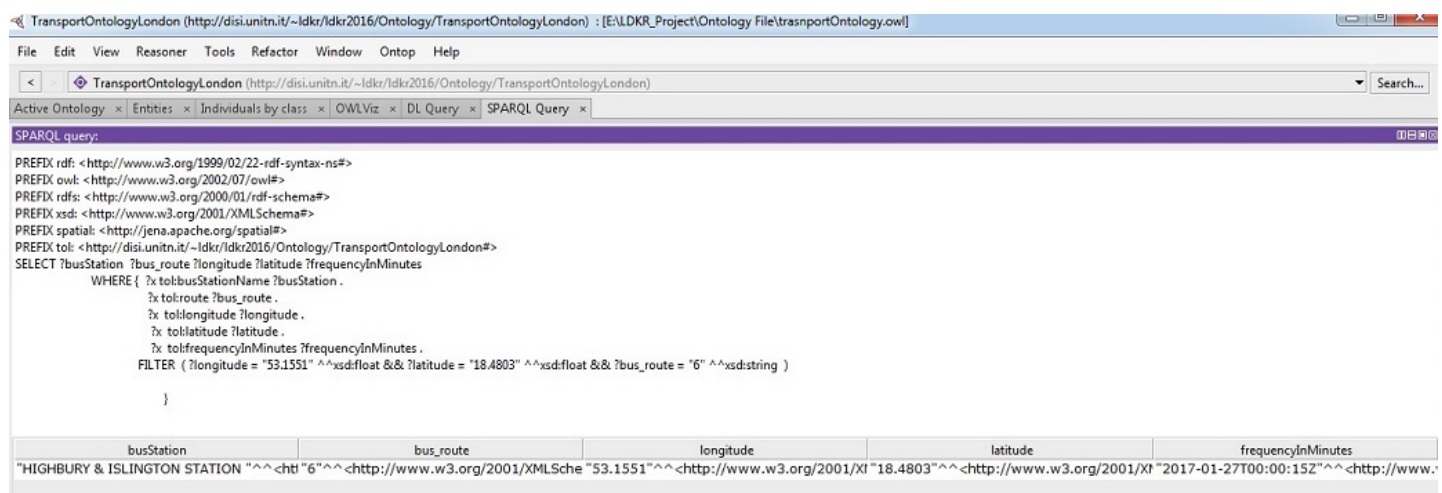

Figure 3: SPARQL Query-At a given bus stop, what is the schedule for buses along with their direction

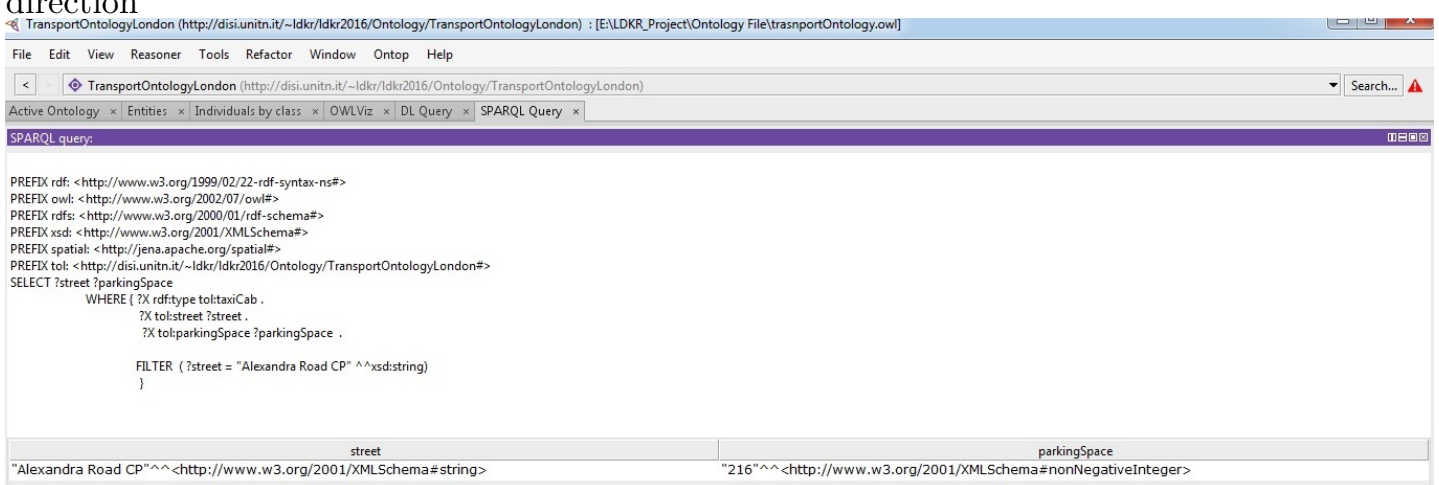

Figure 4: SPARQL Query- What is the parking space availability for a vehicle type at a given street?

\section{Conclusion and Future Work}

In this paper, an ontology for transportation system has been presented, which is developed by understanding the common terms used in the transportation sys- tem. Our ontology is capable of answering the user competence queries. Although our ontology serves the purpose of providing information on transportation sys- tem from a traveler's perspective, it would be noteworthy if it could answer more complex user queries. As a future work, we propose to extend our ontology in order to answer complex competence queries to make the model usable on a large scale. The ontology can be used with data integration technique to build a semantic aware transportation system.

\section{References}

[1] Claude Comtois and Brian Slack. The geography of transport systems. Routledge, 2009.

[2] Marcel Becker and Stephen F Smith. An ontology for multi-modal transportation planning and scheduling. Technical Report CMU-R I-TR-98-15, 1997.

[3] Sabine Timpf. Ontologies of wayfinding: a traveler's perspective. Networks and spatial economics, 2(1):9-33, 2002.

[4] Junli Wang, Zhijun Ding, and Changjun Jiang. An ontology-based public transport query system. In Semantics, Knowledge and Grid, 2005. SKG'05. First International Conference on, pages 62- 
62. IEEE, 2005.

[5] Abolghasem Sadeghi Niaraki and Kyehyun Kim. Ontology based personalized route planning system using a multi-criteria decision making approach. Expert Systems with Applications, 36(2):2250$2259,2009$.

[6] Mnasser Houda, Maha Khemaja, Kathia Oliveira, and Mourad Abed. A public transportation ontology to support user travel planning. In Research Challenges in Information Science (RCIS), 2010 Fourth International Conference on, pages 127-136. IEEE, 2010.

[7] KáThia MarçAl De Oliveira, Firas Bacha, Houda Mnasser, and Mourad Abed. Transportation ontology definition and application for the content personalization of user interfaces. Expert Systems with Applications, 40(8):3145-3159, 2013.

[8] Fausto Giunchiglia and Biswanath Dutta. Dera: A faceted knowledge organization framework. Technical report, University of Trento, 2011.

[9] Fausto Giunchiglia, Biswanath Dutta, and Vincenzo Maltese. From knowledge organization to knowledge representation. Technical report, Università di Trento, 2013.

[10] Subhashis Das and Fausto Giunchiglia. Geoetypes: Harmonizing diversity in geospatial data (short paper). In OTM Confederated International Conferences" On the Move to Meaningful Internet Systems", pages 643-653. Springer, 2016.

[11] Biswanath Dutta, Durgesh Nandini, and Gautam Kishore Shahi. Mod: metadata for ontology description and publication. In International Conference on Dublin Core and Metadata Applications, pages 1-9, 2015.

[12] C Masolo, S Borgo, A Gangemi, N Guarino, A Oltramari, and L Schneider. Dolce: a descriptive ontology for linguistic and cognitive engineering. WonderWeb Project, Deliverable D17 v2, 1:75$105,2003$.

[13] Mark A Musen. The protégé project: a look back and a look forward. AI matters, 1(4):4-12, 2015. 